Journal of Computer Science 5 (4): 255-262, 2009

ISSN 1549-3636

(C) 2009 Science Publications

\title{
MPLS Based Architecture for Mobility and End-to-End QoS Support in Fourth Generation Mobile Networks
}

\author{
Alejandro Quintero and Eduardo Del Frutos \\ Mobile Computing and Networking Research Laboratory, \\ Department of Computer Engineering, École Polytechnique de Montréal Montréal, \\ P.O. Box 6079, Station Centre-Ville, Montréal, Canada H3C3A7
}

\begin{abstract}
Problem statement: Next-generation mobile networks are evolving towards network architectures relying entirely on IP. Approach: These networks had to be scalable in order to support future IP traffic, namely new multimedia services and real time applications, while providing an effective mobility management mechanism to cope with increasingly mobile users. In addition to global connectivity, next-generation mobile networks will have to offer quality of service guarantees such as assured bandwidth, low rate of packet loss, low delay and jitter. Results: In this study, we proposed an MPLS-based architecture for mobility management and end-to-end quality of service support in fourth-generation all-IP mobile networks (MAFI). Conclusion/Recommendations: Our scheme aimed to reduce handoff latency by implementing the fast handover technique and to increase the robustness and flexibility of the mobile system. The results obtained confirm the efficiency of MAFI when compared to FHMIPv6 and others schemes well-known in the literature.
\end{abstract}

Key words: Mobile IP, mobility management, QoS, 4G

\section{INTRODUCTION}

Cellular mobile networks have evolved rapidly in the past few years. At a time when the industry is upgrading its second generation networks to $3 \mathrm{G}$ technology, the research community is looking into fourth generation networks and its challenges. Each generation of networks brings in a wealth of solutions, technologies and a number of new problems and research challenges. Third Generation (3G) mobile systems were designed to support high speed multimedia traffics including data and voice, while fourth Generation (4G) networks will bring wireless systems to the All-IP Net-era. All-IP networks apply the IP technology end-to-end, from the mobile user to the gateway that connects it to the Internet. IPv6, the latest generation of the Internet Protocol, will be the glue that interconnects this heterogeneous world ${ }^{[11]}$. A close look at fourth-generation networks shows that they will provide fully converged services, will offer mobile access in a ubiquitous fashion, will support diverse and heterogeneous user devices, will be autonomous and will depend heavily on software ${ }^{[9]}$.

The latest trend in communications proposes roaming capabilities through different access networks while providing continuous data and voice services. Internet mobility support is even more crucial now than before since many new mobile terminals provide access to IP networks. The advent of UMTS and the various multimedia services it supports implies that user mobility must not only be supported but also well managed.

Supporting Quality of Service (QoS) is a major challenge in $4 \mathrm{G}$ networks. Varying bit rates, channel characteristics, bandwidth allocation, fault-tolerance levels and handoff support among heterogeneous wireless networks make this a difficult task. Adding to this difficulty is the fact that QoS support can occur at various levels ${ }^{[16]}$ :

- Packet-level QoS applies to jitter, throughput and error rate. Network resources such as buffer space and access protocol are likely influenced

- Transaction-level QoS describes both the time it takes to complete a transaction and the packet loss rate. Certain transactions may be time-sensitive, while others cannot tolerate any packet loss

- Circuit-level QoS includes call blocking for new as well as existing calls. It depends primarily on a network's ability to establish and maintain end-to-

Corresponding Author: Alejandro Quintero, Mobile Computing and Networking Research Laboratory,

Department of Computer Engineering, École Polytechnique de Montréal Montréal, P.O. Box 6079, Station Centre-Ville, Montréal, Canada H3C3A7 
end circuits. Call routing and location management are two important circuit-level attributes

- User-level QoS depends on user mobility and application type. The new location may not support the minimum QoS needed, even with adaptive applications $^{[6]}$

In a complete scenario, end-to-end communication between two users will likely involve multiple wireless networks. Since QoS will vary across different networks, the QoS for these users will likely be the minimum level supported the networks. Further work is still needed to fully address end-to-end QoS support as it is still an open issue.

At the highest level, the 4G architecture will include three basic areas of connectivity: Personal Area Networking (PAN) with Bluetooth, local high-speed access points including wireless LAN technologies such as Wi-Fi or hyperlink and cellular connectivity. Under this paradigm, 4G networks involve a wide range of mobile devices that support global roaming. Each device will be able to interact with Internet-based information that can be modified for the network being used by the device at that moment.

Mobility management in IP networks: Mobile IPv6 (MIPv6) ${ }^{[12]}$ was developed by the Internet Engineering Task Force (IETF) to maintain connectivity while users roam through IPv6 networks. Without this protocol, a Mobile Node (MN) cannot receive packets if it roams outside its network. While the MN roams, it must change its IP address each time it crosses a new network. However, all connections above and including the transport layer will be lost since the mobile node's IP address changes each time it moves or changes networks. Indeed, all transport protocols in the TCP/IP family define their connections with the host's and correspondent IP addresses, hence the loss of the contracted connection when one of the addresses changes. IPv6 mobility management is an important aspect of global mobility since it is envisioned that most of the Internet will be populated by IPv6 mobile nodes $^{[9,10,17]}$.

Hierarchical Mobile IPv6 (HMIPv6) ${ }^{[15]}$ complements MIPv6 by facilitating local mobility management. HMIPv6 aims to reduce global signaling and providing improved local mobility management by introducing a hierarchical architecture. Hierarchical mobility management tends to reduce signaling overhead between the mobile node, its correspondent nodes and its home agent. Indeed, by dividing the network in several domains managed by a Mobility
Anchor Point (MAP), a mobile node does not need to update its correspondent nodes when it roams within the same domain. Moreover, by using MAPs, a network is likely to improve MIPv6 handover latency.

Fast Handovers for Mobile IPv6 (FMIPv6) ${ }^{[5]}$ is one of the protocols in IPv6 family that enables a MN to configure a new Care-of-Address (CoA) before it changes network. Thus, the MN can use the CoA right after a connection with the new Access Router (AR) is established. The goal of FMIPv6 is to minimize handover latency since a MN can neither send nor receive packets until the handover completes. Better results are achieved with FMIPv6. However, in high load conditions the amount of signaling degrades its performance. These results are expanded in $^{[11]}$ to include a combination of HMIPv6 and FMIPv6 called Fast handover for Hierarchical Mobile IPv6 (FHMIPv6). Others approaches have been developed for this kind of problem ${ }^{[1,4,8,13,14,18]}$

End-to-end QoS solutions for 4G Networks: Fourthgeneration mobile networks will evolve into all-IP systems, integrating the Internet with mobile wireless systems. This scenario creates new challenges for QoS provision and mobility management, as this new breed of network will have to deal with the fast mobility of terminals regardless of the network access technology or mobility of the terminal. In particular, provision of a wide variety of services in the future mobile Internet demands a technology that combines several kinds of QoS protocols and mobility management techniques. One of the main problems is how to maintain QoS provisioning while the user is roaming.

Shou-Chi Lo et al. ${ }^{[7]}$ propose an architecture which supports both mobility and QoS management in IPbased wireless networks named 'Forwarding Chain and Aggregate Reservation' (FCAR). FCAR is based on the Intra-Domain Management Mobility Protocol (IDMP). In mobility management, the fast handoff, where packets are forwarded to neighboring locations, is used to reduce service disruption. Also, the fast location lookup method, where routing information about a $\mathrm{MN}$ is replicated to several routers, is used to avoid the triangular routing problem incurred by mobile IP. The Resource Reservation Protocol (RSVP) can be also be used to enable end-to-end QoS provisioning. In particular, the RSVP aggregation technique avoids the scalability problem. Moreover, passive resource reservation can be used to reduce the influence of host mobility on the resource reservation delay. QoS provisioning in the access network is based on the IntServ model with a combination of RSVP and RSVP aggregation. 
MPLS is a QoS technology that was introduced to enhance the performance of the internet's datagram model in terms of management and delivery. MPLS is a scalable routing technique where routing is realized by swapping labels on packets instead of the traditional IP destination lookup. A Label Distribution Protocol (LDP) is used to distribute the labels and to maintain the coherence of label bindings across a network. Labels are then used to identify packets through a Label Switched Path (LSP) traversing Label Switching Routers (LSR).

\section{APPROACH}

MAFI: MPLS-based architecture supporting end-toend QoS in 4G mobile networks: We have presented a set of technologies and protocols. These solutions try to conjointly solve the mobility management and QoS provisioning problems in wireless networks. All the proposals use Mobile IPv6 to manage the mobility of users and many of them propose optimizations to improve its operation. Moreover, several of the proposed approaches were based on the use of one of the two QoS paradigms (Integrated Services and Differentiated Services) combined with Mobile IPv6.

We propose a novel architecture to provide End-toEnd QoS in 4G networks. The proposed architecture combines mobility management and QoS provisioning. To manage the mobility, a MIPv6 optimization, called Fast Hierarchical Mobile IPv6, is used. End-to-End QoS is supported through an approach based on a combination of DiffServ and IntServ over MPLS.

A $4 \mathrm{G}$ network can be seen as a set of sub-networks interconnected through IPv6 technology. Integrating the idea of Hierarchical Mobile IPv6 each access router in a particular domain can act as Mobile Anchor Point (MAP) and several hierarchical intra-domain divisions can be realized to improve and optimize user mobility and handoffs.

The next step is to introduce QoS between MNs and their correspondent nodes. The proposed solution integrates HMIPv6 domains in MPLS boundaries. Fig. 1 shows the MAFI architecture. We now define the entities and subsystems in the architecture (Fig. 2).

The RSVP-TE is used for establishing a dynamic LSP. The reservation process is initiated by the MN, in the case of IntServ LSPs or by the GLSR in the case of Signaling and DiffServ LSPs. For IntServ LSPs the MNs indicate the QoS parameters (bandwidth, BER, delay), necessary to communicate with their $\mathrm{CN}$. For inter-domain signaling and DiffServ LSPs, QoS parameters are set up by the GLSR and their value are a function of traffic profiles and the user's number.

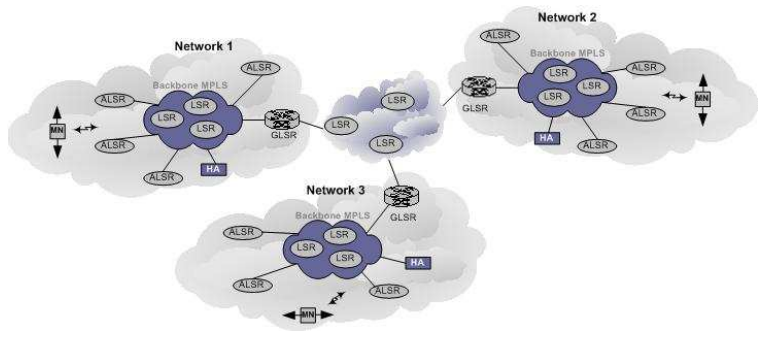

Fig. 1: Proposed MAFI architecture

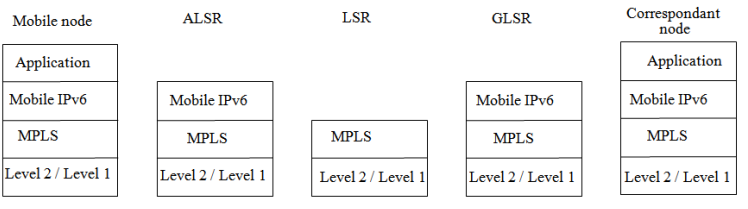

Fig. 2: Protocol architecture of the proposed solution

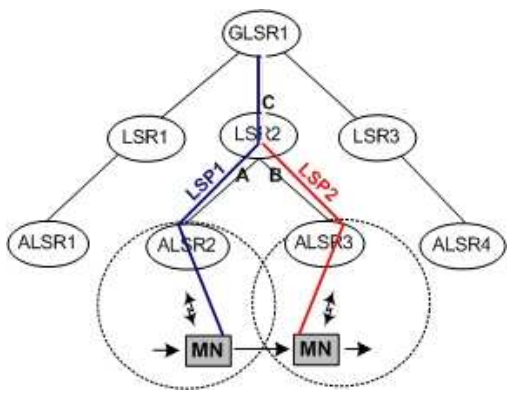

Fig. 3: Path reservation optimization scenario

Mobility and QoS procedures for the IntServ model: As with the conventional Mobile IPv6 procedure, a Mobile Node (MN) located in a foreign network sends its current Regional Care of Address (RCoA) to the Home Agent (HA) by using Binding Update (BU) messages. The Correspondent Node $(\mathrm{CN})$ that wants to establish a QoS session transmits a PATH message (message 1) to the MN's home address since the CN does not know the MN's current location.

Path reservation optimization: The path reservation optimization scenario is shown on Fig. 3. A MN that has established an RSVP session with the GLSR moves to ALSR3 (Access Label Switch router) and initiates the handoff procedure. During this procedure the MN gets a new CoA, actualizes its position and creates a new RSVP path through ALSR3. When the MN moves to ALSR3's cell, it initiates the creation of a new RSVP path by sending a PATH message to ALSR3. This access router forwards the PATH message to LSR2 (Label Switch Router). When LSR2 receives the PATH 
message, it checks if there exists a forwarding entry for the flow identified by the PATH message. An entry in the router table includes the session identifier and the resources reserved for the PATH. A session can be identified by parameters like MN CoA, CN IP address, MN port number.

Inter-domain mobility scenario: In this scenario, the MN moves between two ALSRs that belong to different domains. This procedure is similar to the intra-domain one. First the MN anticipates the handoff using a level 2 trigger. When it receives the network prefix the MN generates a new LCoA (Local Care-of-Address) and sends a S_BU message to GLSR1. The gateway can identify the domain where the MN wants to move by checking the LCoA. Then, it forwards the S_BU (Binding Update) message to the gateway of the new domain, GLSR2, in this case. The message is sent using an inter-domain signaling LSP. If the LSP is not yet created, it must be created by GLSR1 before it can send the message to GLSR2. Afterwards, GLSR2 forwards the S_BU to the access router whose networks prefix coincides with the network prefix of the new LCoA found in the S_BU message.

\section{RESULTS AND DISCUSSION}

Analytical model of the MAFI architecture: we analyze the MAFI architecture and develop an analytical model that will be used to analyze and compare its performances with other architectures found in the literature. Below, we introduce the parameters that will be used in the aforementioned analysis:

\begin{tabular}{|c|c|}
\hline$\lambda_{\mathrm{S}}$ & Average number of session par second \\
\hline$\mu_{\mathrm{S}}$ & Average session connection time \\
\hline$\mu_{R}$ & Average mobile user resident time in a cell \\
\hline$s$ & $\begin{array}{l}\text { Average size of signaling message for } \\
\text { Mobile IPv6 procedures }\end{array}$ \\
\hline $\mathrm{S}_{\mathrm{R}}$ & $\begin{array}{l}\text { Average size of signaling message for QoS } \\
\text { procedures }\end{array}$ \\
\hline P & Average size of a data packet \\
\hline $\mathrm{O}_{\text {MPLS }}$ & ced by MPLS \\
\hline$P_{D}$ & Processing delay in routers \\
\hline$\Omega_{\mathrm{X}, \mathrm{Y}}$ & Average number of hops between $\mathrm{x}, \mathrm{y}$ \\
\hline $\mathrm{B}_{\mathrm{W}}(\mathrm{x}, \mathrm{y})$ & Bandwidth of the wired link $\mathrm{x}, \mathrm{y}$ \\
\hline $\mathrm{B}_{\mathrm{WL}}(\mathrm{x}, \mathrm{y})$ & Bandwidth of the wireless link $x, y$ \\
\hline $\mathrm{D}_{\mathrm{W}}(\mathrm{x}, \mathrm{y})$ & Propagation Delay of the wired link $x, y$ \\
\hline $\mathrm{D}_{\mathrm{WL}}(\mathrm{x}, \mathrm{y})$ & Propagation Delay of the wireless link $\mathrm{x}, \mathrm{y}$ \\
\hline $\mathrm{N}$ & $\begin{array}{l}\text { Average number of movements during a } \\
\text { session }\left(\mathrm{Nm}=\mu_{\mathrm{S}} \mu_{\mathrm{R}}^{-1}\right)\end{array}$ \\
\hline
\end{tabular}

CN Average number of correspondent nodes during a session

$\mathrm{H} \quad$ Number of wired links

Let TTX (s, x, y) denote the transmission delay of a message of size $S_{p}$ sent from $x$ to $y$. TTX (s, $\left.x, y\right)$ can be expressed as follows:

$$
\begin{aligned}
\mathrm{T}_{\mathrm{TX}}\left(\mathrm{s}_{\mathrm{p}}, \mathrm{x}, \mathrm{y}\right)= & \left(\frac{\mathrm{s}_{\mathrm{p}} \cdot \mathrm{O}_{\mathrm{MPLS}}}{\mathrm{B}_{\mathrm{wL}}(\mathrm{MN}, \mathrm{ALSR})}+\mathrm{D}_{\mathrm{wL}}(\mathrm{MN}, \mathrm{ALSR})\right) \\
& +\sum_{\mathrm{i}=1}^{\mathrm{H}-2} \sum_{\mathrm{j}=1}^{\mathrm{H}-2}\left(\frac{\mathrm{s}_{\mathrm{p}} \cdot \mathrm{O}_{\mathrm{MPLS}}}{\mathrm{B}_{\mathrm{w}}(\mathrm{i}, \mathrm{j})}+\mathrm{D}_{\mathrm{w}}(\mathrm{i}, \mathrm{j})\right)+\left(\Omega_{\mathrm{MN}, \mathrm{CN}}+1\right) \cdot \mathrm{P}_{\mathrm{D}}
\end{aligned}
$$

Supposing constant bandwidth and delay in wired links, equal to $\mathrm{BW}$ and $\mathrm{DW}$ respectively, the equation simplifies to:

$$
\begin{aligned}
\mathrm{T}_{\mathrm{TX}}\left(\mathrm{s}_{\mathrm{p}}, \mathrm{x}, \mathrm{y}\right)= & \left(\frac{\mathrm{s}_{\mathrm{p}} \cdot \mathrm{O}_{\mathrm{MPLS}}}{\mathrm{B}_{\mathrm{WL}}(\mathrm{MN}, \mathrm{ALSR})}+\mathrm{D}_{\mathrm{wL}}(\mathrm{MN}, \mathrm{ALSR})\right) \\
& +\Omega_{\mathrm{MN}, \mathrm{CN}} \cdot\left(\frac{\mathrm{s}_{\mathrm{P}} \cdot \mathrm{O}_{\mathrm{MPLS}}}{\mathrm{B}_{\mathrm{W}}}+\mathrm{D}_{\mathrm{w}}\right)+\left(\Omega_{\mathrm{MN}, \mathrm{CN}}+1\right) \cdot \mathrm{P}_{\mathrm{D}}
\end{aligned}
$$

The results obtained for our architecture will be compared with two solutions referenced in the literature:

Architecture for Mobility and QoS Support in All-IP Wireless Networks ${ }^{[7]}$. This solution will be named FCAR:

- RSVP extension for HMIPv6 environments with Fast Handoffs ${ }^{[2]}$. This solution will be named FHRSVP.

- To simplify comparisons with these solutions, all the calculus will be done over the architecture shown on Fig. 3.

Signaling cost: The total signaling cost of registration updates during a session is denoted by $C_{R}$. The signaling cost is defined as the number of bytes sent by the $\mathrm{MN}$ in order to complete the handoff procedure during a session.

The signaling cost can be calculated as the sum of two terms:

- The cost associated with the registration update and MobileIPv6 procedures $\left(\mathrm{C}_{\mathrm{MIP}}\right)$

- The cost associated with the QoS reservation procedures $\left(\mathrm{C}_{\mathrm{Qos}}\right)$

The signaling cost of our proposal will be compared with the other two solutions. 


\section{Intra-domain signaling cost: \\ MAFI:}

$$
\begin{aligned}
& \mathrm{C}_{\mathrm{MIP}}=\mathrm{s}_{\mathrm{S}} \cdot\left(\Omega_{\mathrm{MN}, \mathrm{GLSR}}+\Omega_{\mathrm{GLSR}, \mathrm{ALSR}}+\sum^{\text {NoCN }} 2 \cdot \Omega_{\mathrm{MN}, \mathrm{GLLSR}} \cdot \mathrm{p}_{\mathrm{CN}}\right) \cdot \mathrm{N}_{\mathrm{M}} \\
& \mathrm{C}_{\mathrm{QoS}}=2 \cdot \mathrm{s}_{\mathrm{R}} \cdot\left(\begin{array}{l}
\Omega_{\mathrm{GLSR}, \mathrm{ALSR}}+\Omega_{\mathrm{ALSR}, \mathrm{MN}}+ \\
\left(1-\mathrm{p}_{\mathrm{Path}}\right) \cdot \Omega_{\mathrm{MN}, \mathrm{ALSR}}+\mathrm{p}_{\mathrm{Path}} \cdot \mathrm{r} \cdot \Omega_{\mathrm{MN}, \mathrm{ALSR}}
\end{array}\right) \cdot \mathrm{N}_{\mathrm{M}}
\end{aligned}
$$

Where:

$\mathrm{p}_{\text {path }}=$ Probability of the path reservation optimization occurs

$r=$ Cost reduction if the path reservation optimization occurs

$\mathrm{p}_{\mathrm{CN}}=$ Probability of correspondent $\mathrm{i}$ resides in the same domain

\section{FCAR:}

$\mathrm{C}_{\mathrm{MIP}}=2 \cdot \mathrm{s}_{\mathrm{S}} \cdot\left(2 \cdot \Omega_{\mathrm{MN}, \mathrm{ALSR}}+\Omega_{\mathrm{ALSROd}, \mathrm{ALSRRew}}+\sum^{\text {NoCN }} 2 \cdot \Omega_{\mathrm{MN}, \mathrm{GLSR}} \cdot \mathrm{p}_{\mathrm{CN}}\right)$

$\cdot \mathrm{N}_{\mathrm{M}}+2 \cdot \mathrm{s}_{\mathrm{S}} \cdot \Omega_{\mathrm{MN}, \mathrm{ALSR}} \cdot \mathrm{N}_{\mathrm{r}}$

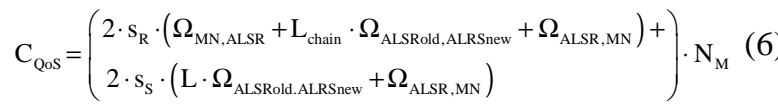

Where:

$\mathrm{L}_{\text {chain }}=$ Length of forwarding chain

$\mathrm{N}_{\mathrm{r}}$ = Average number of renewals of the forwarding chain during a session

\section{FH-RSVP:}

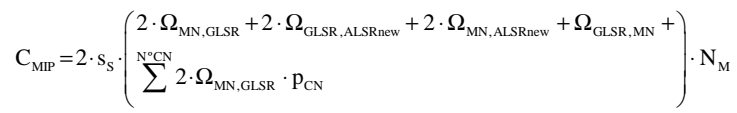

$$
\begin{aligned}
& \mathrm{C}_{\mathrm{QOS}}=4 \cdot \mathrm{s}_{\mathrm{R}} \cdot \Omega_{\mathrm{MN}, \mathrm{GLRS}} \cdot \mathrm{N}_{\mathrm{M}}
\end{aligned}
$$

Average signaling cost: The average signaling cost for each solution can be calculated according to the next formula:

$$
\mathrm{C}_{\mathrm{T}}=\left(\mathrm{C}_{\mathrm{MIP}_{\text {_intra }}}+\mathrm{C}_{\text {Qos__intra }}\right) \cdot \mathrm{p}_{\text {intra }}+\left(\mathrm{C}_{\mathrm{MIP}_{\text {Inter }}}+\mathrm{C}_{\text {Qos_inter }}\right) \cdot \mathrm{p}_{\text {inter }}
$$

The values in Table 1 are used to compare the three handoff mechanisms that were modeled.

We assume that the average residence time in a cell for a mobile user varies between 1-100 sec. Figure 4 shows the average signaling cost for MAFI, FCAR, FHRSVP.
Table 1: Values for the average handoff time analysis

\begin{tabular}{ll}
\hline Parameter & Value \\
\hline Pintra & 0.7 \\
Pinter & 0.3 \\
Average size MIP message & 38 bytes \\
Average size QoS message & 48 bytes \\
$\begin{array}{l}\text { Overload MPLS } \\
\text { Probability of the correspondent node resides in } \\
\text { the same domain }\end{array}$ & 4 bytes \\
Probability of a path reservation optimization occurs & 0.5 \\
$\begin{array}{l}\text { Reduction as consequence of a path reservation } \\
\text { optimization }\end{array}$ & 0.5 \\
Number of Correspondent Nodes $(\mathrm{CN})$ & 0.5 \\
$\mu_{\mathrm{s}}$ & 10 \\
\hline
\end{tabular}

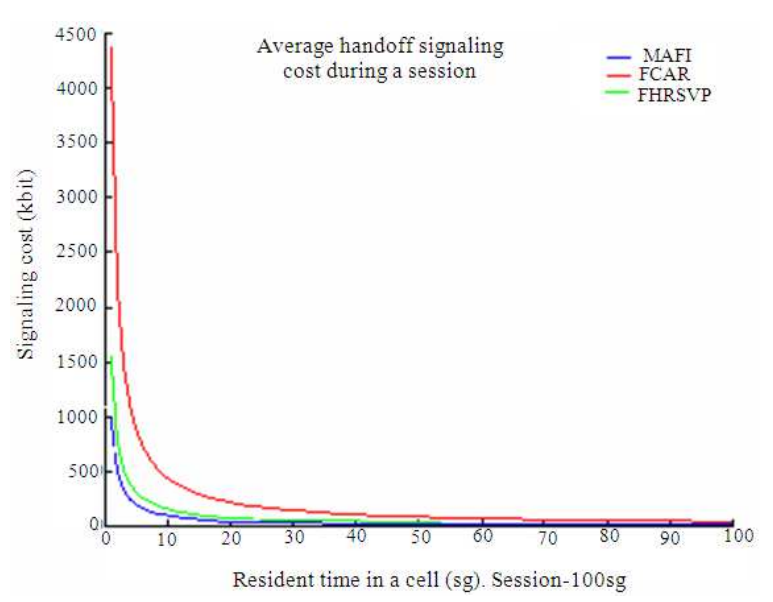

Fig. 4: Average signaling cost during a session. Resident time in a cell variable

Blocking probability: Let $A_{\text {sig }}=2 \cdot \frac{T_{\text {handoff }} \cdot \sigma \cdot \pi R^{2} \cdot \beta}{2 \mu_{R}}$ be the total signaling traffic load in a cell and assuming the next values:

- Average mobile user resident time $\left(\mu_{R}\right)$ in a cell varies between 1-100 sec

- Average session connection time $\left(\mu_{\mathrm{s}}\right): 100 \mathrm{sec}$

- Number of mobile users that move to another cell $(\beta): 0.25$

- Number of signaling channels $(\mathrm{N})$ varies between 60-80

- Density of mobile users ( $\sigma): 0.25$ mobile users $\mathrm{m}^{-2}$

- Cells radius (R): $250 \mathrm{~m}$

The signaling blocking probability, with $60-80$ channels, is shows in Fig. 5.

Simulation results: We simulate MAFI and compare its performance with FCAR and FH-RSVP. We used the NS 2.27 simulator. All the simulations were done over the scenario shows on Fig. 6. These scenarios coincide with the scenarios used in the analytical study done. 
An 11 Mbps $802.11 \mathrm{~b}$ wireless LAN was used for the wireless access network. The wired zone and the backbone were configured with links of 50 and $100 \mathrm{Mbps}$ and delays of 10 and $4 \mathrm{~m} \mathrm{sec}$, respectively.

Three mobility scenarios have been studied Fig. 79. In the first scenario, shown on Fig. 7, the MN moves across the four radio coverage areas (from ALSR1 to ALSR4) residing in each area for a short amount of time.

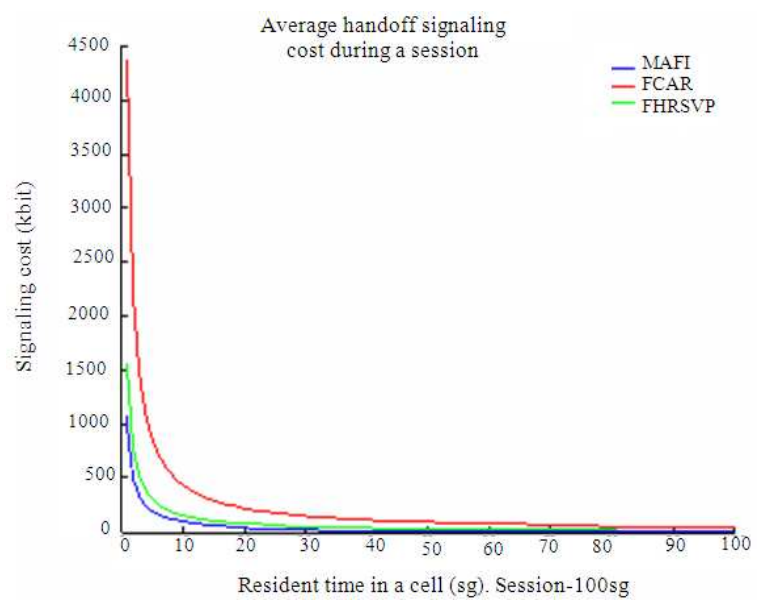

Fig. 5: Signaling blocking probability with 60 channels

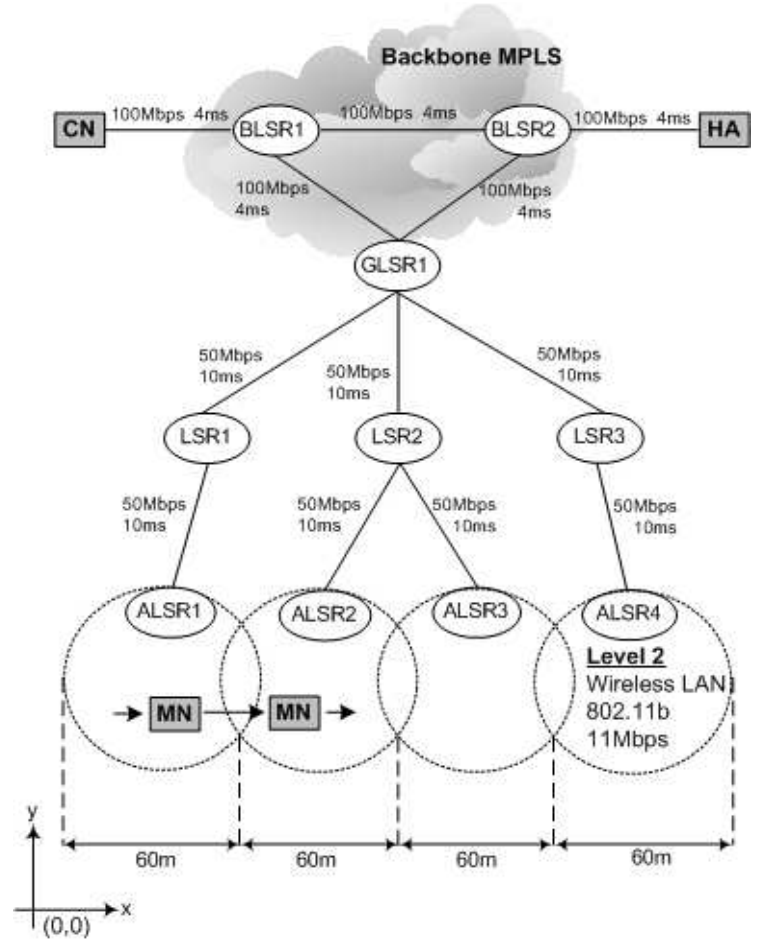

Fig. 6: General scenario used in simulations
In the second scenario, shown on Fig. 8, the mobile node goes directly from ALSR1 to ALSR4.

In the last scenario, shown on Fig. 9, a back and forth movement between ALSR1 and ALSR2 was studied.

Each scenario was tested by varying the speed of the mobile node. Several speeds varying from 5$70 \mathrm{~m} \mathrm{sec}^{-1}$ were simulated. Two traffic flows were defined for communication between a mobile node and its correspondent node:

- Constant Bit Rate (CBR) traffic over UDP. This traffic flow tries to emulate the typical conversational and streaming applications (real time video, streaming video). A bit rate of $400 \mathrm{kbps}$ was defined for all simulations

- $\quad$ FTP (over TCP) traffic. With this kind of traffic we study TCP performance in a mobile environment. We used TCP Reno

Latency: During the simulation, several handoffs occurred. For example, Table 2 shows handoffs that occurred for scenario 1 when the $\mathrm{MN}$ move at $10 \mathrm{~m} \mathrm{sec}^{-1}$.

In the results shown in Table 2, we can clearly see the exact time and the position of the mobile node when the handoff occurs. Furthermore, we can see the access router (ALSR) that uses the mobile node to send and receive data packets. In the case of FHMIP/MPLS,

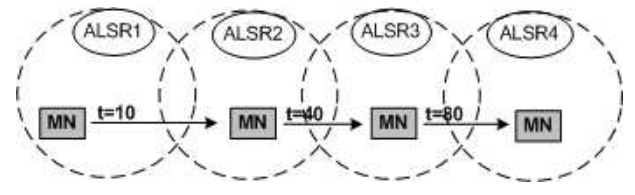

Fig. 7: Scenario 1

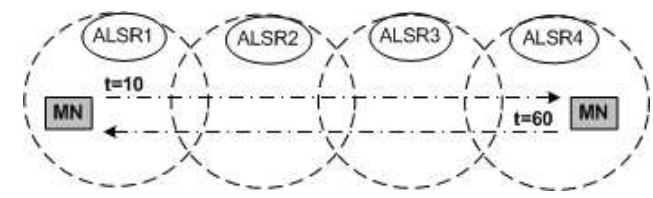

Fig. 8: Scenario 2

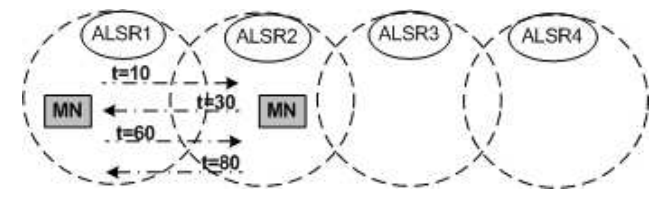

Fig. 9: Scenario 3 
J. Computer Sci., 5 (4): 255-262, 2009

Table 2: Handoff results for scenario 1 when the MN moves at a. speed of $10 \mathrm{~m} \mathrm{sec}^{-1}$

\begin{tabular}{|c|c|c|c|c|c|c|c|c|c|}
\hline $\begin{array}{l}\text { FHMIP/ } \\
\text { MPLS } \\
\text { Event time }\end{array}$ & $\begin{array}{l}\mathrm{v}=10 \mathrm{~m} \mathrm{sec}^{-1} \\
\text { Handoff } \\
\text { delay }(\mathrm{sec})\end{array}$ & ALSR & $\begin{array}{l}\text { MN } \\
\text { position } \\
\mathrm{X} \\
\end{array}$ & $\begin{array}{l}\text { MN } \\
\text { position } \\
\mathrm{Y}\end{array}$ & $\begin{array}{l}\text { MAFI } \\
\text { Event time }\end{array}$ & $\begin{array}{l}\mathrm{v}=10 \mathrm{~m} \mathrm{sec}^{-1} \\
\text { Handoff } \\
\text { delay }(\mathrm{sec})\end{array}$ & ALSR & $\begin{array}{l}\text { MN } \\
\text { position } \\
\mathrm{X} \\
\end{array}$ & $\begin{array}{l}\text { MN } \\
\text { position } \\
\mathrm{Y}\end{array}$ \\
\hline 14,3160912 & 0,015 & ALSR2 & 141,91 & 53,1 & 11,5140912 & 0,004 & ALSR2 & 114,91 & 57,52 \\
\hline 42,2880912 & 0,002 & ALSR3 & 182,87 & 50 & 42,3640912 & 0,002 & ALSR3 & 183,63 & 50 \\
\hline 42,3120912 & 0,002 & ALSR2 & 183,11 & 50 & & & & & \\
\hline 43,8780912 & 0,009 & ALSR3 & 198,27 & 50 & & & & & \\
\hline 82,2480912 & 0,002 & ALSR4 & 242,47 & 50 & 82,3240912 & 0,002 & ALSR4 & 243,23 & 50 \\
\hline 82,2800912 & 0,002 & ALSR3 & 242,79 & 50 & & & & & \\
\hline 82,3280912 & 0,004 & ALSR4 & 243,27 & 50 & & & & & \\
\hline
\end{tabular}

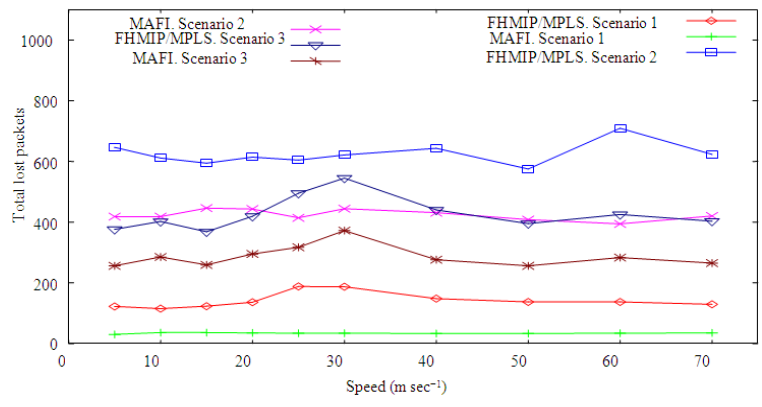

Fig. 10: CBR total lost packets

the handoff event has a transitory time in the overlapping zones. In these zones, the mobile node alternates the use of access routers from which it receives a signal. The $\mathrm{MN}$ selects the most suitable access router based on the power levels received from each access router.

The average handoff latency obtained in simulations is:

- MAFI: $2.6 \mathrm{~m} \mathrm{sec}^{-1}$

- FHMIP/MPLS: $5.1 \mathrm{~m} \mathrm{sec}^{-1}$

Lost packets: Figure 10 shows the total lost packets for each mobile node speed. We can clearly see that in all scenarios MAFI loses fewer packets than its FHMIP/MPLS counterpart. Indeed, the optimizations we incorporated to FHMIPv6 over MPLS substantially reduced the number of lost packets.

The best performances are found in scenario 1 when the mobile node roams from ALSR1 to ALSR4 and stops in each cell in-between. The packet loss rates for MAFI are on average a little over 50 packets while they amount to more than 150 packets for FHMIP/MPLS. The worst performances are found, without surprise, in scenario 2 where the mobile roams directly from ALSR1 to ALSR4 without stopping in adjacent cells. In this worst-case scenario, MAFI loses on average no more than 425 packets while the number of packets lost for FHMIP/MPLS exceeds 600. Overall, for the three pairs of curves, MAFI improves packet loss rates by more that $30 \%$ when compared to FHMIP/MPLS.

\section{CONCLUSION}

In this study, we have proposed an End-to-End QoS architecture based on FHMIPv6 and MPLS for 4G mobile networks (MAFI). The goal of this work was to define a set of procedures and mechanisms that can be applied on a $4 \mathrm{G}$ mobile network architecture.

Our proposal integrates the advantages of these proposals and resolves several limitations that were not considered in the individual solutions. MAFI propose an adaptive End-to-End QoS provisioning architecture that integrates mobility management procedures for intra and inter-domain mobility. FHMIPv6 manages the MN's mobility while a combination of DiffServ and Intserv is used for providing specific QoS levels. DiffServ is used for applications without strict QoS restrictions (web browsing, background applications) while IntServ is used to satisfy traffics such as VoIP, real-time applications, streaming, requiring specific QoS values. The necessary resources for each application are reserved along the path between sender and receiver in order to ensure a specific End-to-End QoS level. We modified the RSVP-TE standard in order to improve its efficiency in mobile environments. These modifications reduce the handoff delay and the total signaling cost related to mobility events.

Our solution is compared with FCAR and FHRSVP. This comparison is based on mathematical models in MATLAB and tested over several scenarios. Handoff delay, signaling cost, lost packets and signaling channel blocking probability are evaluated and compared. Analytical results show that MAFI has the lowest signaling cost and lost packets ratio when compared with FCAR and FH-RSVP and is surpassed only by FCAR in handoff delay and signaling channel blocking probability.

We have compared our proposal with FHMIPv6 over MPLS using the NS-2 simulator. We analyzed the response of two traffic sources, namely CBR and TCP, to mobility events and a new estimation of the handoff 
delay was calculated. Three mobility scenarios were defined where the MN moves at different speeds. For the CBR traffic, the response time of MAFI is always better that the one obtained for FHMIPv6 over MPLS. In the case of TCP, the response time is better when the $\mathrm{MN}$ moves at speeds lower than $40 \mathrm{~m} \mathrm{sec}^{-1}$. For speeds above $40 \mathrm{~m} \mathrm{sec}^{-1}$, the response times for MAFI and FHMIPv6/MPLS are similar.

\section{REFERENCES}

1. Albertengo, G., C. Pastrone and G. Tolu, 2005. MOON: A new overlay network architecture for mobility and QoS support. Next Generat. Internet Networks, 19 : 458-465. DOI: 10.1109/NGI.2005.1431701

2. Elleingand, S. and S. Pierre, 2007. FH-RSVP scheme for intra-site handover in hierarchical mobile IPv6 networks. Comput. Commun., 30: 416-427. http://portal.acm.org/citation.cfm?id=1223561

3. Hui, S.Y. and K.H. Yeung, 2003. Challenges in the migration to $4 \mathrm{G}$ mobile systems. IEEE Commun. Mag., 41: 54-59. DOI: 10.1109/MCOM.2003.1252799

4. Kalebaila, G. and A. Chan, 2006. Advanced mobility support in next-generation all-IP wireless networks: A cross-layer approach. Proceedings of the IEEE 17th International Symposium on Personal, Indoor and Mobile Radio Communications, Sept. 11-14, IEEE Xplore Press, Helsinki, pp: 1-5. DOI: 10.1109/PIMRC.2006.254234

5. Rajeev Koodli, 2005. Fast Handovers for Mobile IPv6. IETF RFC4068. http://www.ietf.org/rfc/rfc4068.txt

6. Kumar, A., B.R. Badrinath and A. Acharya, 2001. MRSVP: A resource reservation protocol for an integrated services network with mobile hosts. Wireless Networks, 7: 5-19. http://portal.acm.org/citation.cfm?id=376679.376689

7. Lo, S.C., G. Lee, W.T. Chen and J.C. Lui, 2004. Architecture for mobility and QoS support in all-IP wireless networks. IEEE J. Selected Areas Commun., 22: 691-705. DOI: 10.1109/JSAC.2004.825964

8. Misra, I.S., M. Chakraborty, D. Saha and A. Mukherjee, 2006. An approach for optimal hierarchical mobility, management network architecture. Proceeding of the IEEE 63rd Conference on Vehicular Technology, pp: 481-485. DOI: 10.1109/VETECS.2006.1682861
9. Montavont, N. and T. Noël, 2002. Handover management for mobile nodes in IPv6 networks. IEEE Commun. Mag., 40: 38-43. DOI: 10.1109/MCOM.2002.1024413

10. Omae, K., T. Ikeda, M. Inoue, I. Okajima and N. Umeda, 2002. Mobile node extension employing buffering function to improve handoff performance. Proceedings of the 5th International Symposium on Wireless Personal Multimedia Communications, pp: 62-66. DOI: 10.1109/WPMC.2002.1088132

11. Perez, X., M. Torrent and H. Hartenstein, 2003. A performance comparision of mobile IPv6, hierarchical mobile IPv6, fast handovers for mobile IPv6 and their combination. ACM SIGMOBILE Mobile Comput. Commun. Rev., 7: 5-19. http://portal.acm.org/citation.cfm?id=965736

12. Johnson, D., C. Perkins and J. Arkko, 2004. Mobility Support in Ipv6. IETF RFC 3775. http://tools.ietf.org/html/rfc3775

13. Petander, H., E. Perera, K.C. Lan and A. Seneviratne, 2006. Measuring and improving the performance of network mobility management in IPv6 networks. IEEE J. Select. Areas Commun., 24: 1671-1681. DOI: $10.1109 /$ JSAC.2006.875116

14. Qiang, L. and Z. Ping, 2006. A novel mobility management architecture based on GLL in configurable networks. Proceedings of the 6th International Conference on ITS Telecommunications, June 2006, pp: 70-73. DOI: 10.1109/ITST.2006.288763

15. Hesham, S., C. Flarion, E. Karim and L. Bellier, 2005. Hierarchical mobile IPv6 mobility management (HMIPv6). IETF RFC 4140. http://tools.ietf.org/html/rfc5380

16. Upkar, V. and J. Radhika, 2001. Issues in emerging 4G wireless networks. ACM Comput., 34: 94-96.

17. Vaughan-Nichols, S., 2003. Mobile IPv6 and the future of wireless internet access. ACM Comput., 36: 18-20. DOI: 10.1109/MC.2003.1178041

18. Wang, C. and C. Chen, 2006. An overlay network architecture for MPLS-based, micro-mobility with label stack support. Proceedings of the IEEE 63rd Vehicular Technology Conference, May 2006, IEEE Computer Society, Washington DC., USA., pp: 486-490. DOI: 10.1109/ VETECS.2006.1682862 